CARPATHIAN J. MATH.

Volume 37 (2021), No. 1,

Pages 109 - 117
Online version at https : //www . carpathian. cunbm . utcluj. ro/

Print Edition: ISSN 1584 - 2851; Online Edition: ISSN 1843 - 4401

DOI: https://doi.org/10.37193/CJM.2021.01.11

\title{
Gauge strongly summability of measurable functions
}

\author{
RABIA SAVAŞ and RICHARD F. PATTERSON
}

\begin{abstract}
During the late 50's and early 60's, the notion of Gauge integral was presented by Kurzweil and Henstock, independently. The purpose of this paper is to extend this concept to Summability theory. To accomplish this, we introduce the notion of $\widetilde{\gamma}$-strongly summable to $L$ with respect to Gauge by using $h(\vartheta)$ measurable real valued function defined on $(1, \infty)$. We shall also prove inclusion theorems to contrast it with other Summability integration techniques.
\end{abstract}

\section{INTRODUCTION, PRELIMINARIES AND DEFINITIONS}

In 1957, Kurzweil in [3] introduced the concept of Gauge integral that is more general than Lebesgue's integral. Following, this approach was discovering independently by Henstock [2]. Henstock named such generalization as "Gauge theory". This concept conserves the intuitive appearance of Riemann's definition of the integral, yet has the power of Lebesgue's definition. Although the Henstock-Kurzweil formulation is a small change of the Riemann integral, Gauge integral presumably has the strongest convergence theorems of any integral. The fundamental premise is to apply the standard $\delta$, introduction of the Riemann integral with only one modification, replacing the constant $\delta$ with a function. This function, which was denoted by $\gamma$, is called a Gauge, and it stands for an open interval that differs in length. This small variance has enormous influence in applications such as moduli spaces, topological invariants and quantum aspects. Please observe the following containments.

$$
\left\{\begin{array}{c}
\text { Riemann } \\
\text { integrable } \\
\text { functions }
\end{array}\right\} \subset\left\{\begin{array}{c}
\text { Lebesgue } \\
\text { integrable } \\
\text { functions }
\end{array}\right\} \subset\left\{\begin{array}{c}
\text { Gauge } \\
\text { integrable } \\
\text { functions }
\end{array}\right\}
$$

for proper integrals. The classes of Gauge integrable functions and Lebesgue integrable functions are closely related. We consider only integrals of functions from $[c, d]$ to $\mathbb{R}$, and every improper Riemann integral is not a Lebesgue integral. As illustrated by the following example, $h(r)=r^{2} \cos \left(\frac{1}{r^{2}}\right)$ with $h(0)=0$. Then $\int_{0}^{1}\left|h^{\prime}(r)\right| d r$ is infinite and Lebesgue integral $\int_{0}^{1} h^{\prime}(r) d r$ does not exist, but improper Riemann $\int_{0}^{R} h^{\prime}(r) d r$ exists and equals $h(R)$, and also every Lebesgue integrable and improper Riemann integrable function is Gauge integrable. For the most part, the big advantage of the Gauge integral is the new insight that it yields into the Lebesgue theory. Now, let us present the concepts of the Gauge that are the basis for Gauge theory as follows:

Received: 12.02.2020. In revised form: 14.11.2020. Accepted: 21.11.2020

2010 Mathematics Subject Classification. 40F05, 40G05.

Key words and phrases. Gauge integration, strongly summability, measurable functions.

Corresponding author: Rabia Savas; rabiasavass@hotmail.com 
Definition 1.1. [5] A tagged partition of an interval $J=[c, d]$ is a finite set or ordered pairs

$$
D=\left\{\left(\omega_{i}, J_{i}\right): 1 \leq i \leq p\right\}
$$

where $\left\{J_{i}: 1 \leq i \leq p\right\}$ is a partition of $J$ comprising of closed non overlapping subintervals and $\omega_{i}$ is a point that belongs to $J_{i} ; \omega_{i}$ is named as the tag related to $J_{i}$. If $h: J \rightarrow \mathbb{R}$, the Riemann sum of $h$ in regard to $D$ is defined as follows

$$
S(h, D)=\sum_{i=1}^{p} h\left(\omega_{i}\right) \ell\left(J_{i}\right)
$$

where $\ell\left(J_{i}\right)$ is the length of the subinterval $J_{i}$. If $\delta: J \rightarrow(0, \infty)$ is a positive function, we define an open interval valued function on $J$ by stating $\bar{\gamma}(\omega)=(\omega-\delta(\omega), \omega+\delta(\omega))$. If $J_{i}=\left[y_{i}, y_{i+1}\right]$, then we can write $\omega_{i} \in J_{i} \subset \bar{\gamma}\left(\omega_{i}\right)$ instead of $\omega_{i}-\delta<y_{i} \leq \omega_{i} \leq y_{i+1}<\omega_{i}+\delta$. Any interval $\bar{\gamma}$ defined on $J$ such that $\bar{\gamma}(\omega)$ is an open interval containing $\omega$ for each $\omega \in J$ is called a Gauge on $J$. The set of all such intervals will be denoted by $\Delta_{G}$. If $D=\left\{\left(\omega_{i}, J_{i}\right): 1 \leq i \leq p\right\}$ is a tagged partition of $J$ and $\bar{\gamma}$ is a Gauge on $J$, we say that $D$ is $\bar{\gamma}-$ fine if $\omega_{i} \in J_{i} \subset \bar{\gamma}\left(\omega_{i}\right)$ is fullfilled.

Example 1.1. Let us consider the interval $[0,1]$, and

$$
\bar{\gamma}(y)= \begin{cases}1 / 4 & \text { for } y=0 \\ y / 3 & \text { for } 0<y \leq 1\end{cases}
$$

Also, we consider the following partition by selecting the first tag from the initial interval to become 0 and each tag from every other interval to be the right and end-point of that interval:

$$
\begin{aligned}
& m\left(\left[0, \frac{1}{5}\right]\right)<\frac{1}{5}<\gamma(0)=\frac{1}{4}, m\left(\left[\frac{1}{5}, \frac{1}{4}\right]\right)<\frac{1}{20}<\gamma\left(\frac{1}{4}\right)=\frac{1}{12}, m\left(\left[\frac{1}{4}, \frac{1}{3}\right]\right)<\frac{1}{12}<\gamma\left(\frac{1}{3}\right)=\frac{1}{9}, \\
& m\left(\left[\frac{1}{3}, \frac{2}{5}\right]\right)<\frac{1}{15}<\gamma\left(\frac{2}{5}\right)=\frac{2}{15}, m\left(\left[\frac{2}{5}, \frac{1}{2}\right]\right)<\frac{1}{10}<\gamma\left(\frac{1}{2}\right)=\frac{1}{6}, m\left(\left[\frac{1}{2}, \frac{3}{5}\right]\right)<\frac{1}{10}<\gamma\left(\frac{3}{5}\right)=\frac{1}{5}, \\
& m\left(\left[\frac{3}{5}, \frac{3}{4}\right]\right)<\frac{3}{20}<\gamma\left(\frac{3}{4}\right)=\frac{1}{4}, m\left(\left[\frac{3}{4}, 1\right]\right)<\frac{1}{4}<\gamma(1)=\frac{1}{3} .
\end{aligned}
$$

This is an instance of a $\bar{\gamma}(y)$ - fine tagged partition.

Let us now present the definition of Gauge integral written below:

Definition 1.2. [5] Let $h:[c, d] \rightarrow \mathbb{R}$. Provided that there exist $A \in \mathbb{R}$ such that for every $\varepsilon>0$ and a Gauge $\bar{\gamma}$ on $[c, d]$ such that $|S(h, D)-A|<\varepsilon$ whenever $D$ is a $\bar{\gamma}-$ fine tagged partition of $[c, d]$, then $h$ is said to be Gauge integrable over $[c, d]$. The number $A$ is called the Gauge integral of $h$ over $J=[c, d]$ and is denoted by $\int_{c}^{d} h$ or $\int_{J} h$; when we see integrals depending upon parameters, it is also proper to write $\int_{c}^{d} h(\omega)$ or $\int_{J} h(\omega)$.

Example 1.2. The Dirichlet function:

$$
h(y)= \begin{cases}1, & \text { if } y \in \mathbb{Q} \\ 0, & \text { if } y \in \mathbb{R} / \mathbb{Q} .\end{cases}
$$

is gauge integrable over $[c, d]$. Let $\varepsilon>0$ and let $\left\{r_{l}\right\}$ be an enumeration of the rational numbers in $[c, d]$. Define a Gauge $\delta$ on $[c, d]$ by

$$
\delta(\omega)= \begin{cases}\frac{\varepsilon}{2^{l}}, & \omega=r_{l} \\ 1, & \omega \notin \mathbb{Q} .\end{cases}
$$


If $\bar{P}$ is a $\delta$-fine tagged partition of $[c, d]$, we can separate $\bar{P}$ into $P_{r}$, those tagged intervals with rational tags, and $P_{s}$ with irrational tags. Therefore,

$$
\begin{aligned}
|S(h, \bar{P})-0| & =\sum_{\bar{P}} h\left(\omega_{l}\right) \cdot \Delta y_{l} \\
& =\sum_{P_{r}} 1 \cdot \Delta y_{l}+\sum_{P_{s}} 0 \cdot \Delta y_{l} \\
& <\sum_{n} \frac{\varepsilon}{2^{n}}=\varepsilon .
\end{aligned}
$$

Hence, the Dirichlet function is Gauge integrable with $\int_{c}^{d} h=0$.

Example 1.3. Let us consider the function $h:[0,1] \rightarrow \mathbb{R}$ defined by

$$
h(y)= \begin{cases}\frac{1}{\sqrt{y}}, & \text { if } y>0 \\ 0, & \text { if } y \leq 0\end{cases}
$$

and when we ensure that a function is Gauge integrable, we will replace the value of the integral by such a telescoping sum. $h$ is Gauge integrable over $[0,1]$ with $\int_{0}^{1} h=2$. To confirm this integral, let $0<\varepsilon \leq 1$. A choice of Gauge $\bar{\gamma}$ that will ensure $|S(h, D)-2|<\varepsilon$ for all $\bar{\gamma}-$ fine tagged partitions $D$ of $[0,1]$ is

$$
\gamma(\omega)= \begin{cases}\left(\omega \cdot a^{2}(\omega), \frac{1}{a^{2}(\omega)}\right), & \text { if } \omega>0 \\ \left(-\varepsilon^{2}, \varepsilon^{2}\right), & \text { if } \omega=0\end{cases}
$$

where $0<\alpha(\omega)<1$. Suppose that $\bar{P}=\left\{\left(\omega_{l},\left[y_{l-1}, y_{l}\right]\right)\right\}_{l=1}^{m}$ is a $\bar{\gamma}$-fine tagged partition of $[0,1]$. Then, for $l>1, \frac{2}{\sqrt{y_{l}}+\sqrt{y_{l-1}}}$, and $\frac{1}{\sqrt{\omega_{l}}}$ both fall in the interval $\left[\frac{1}{\sqrt{y_{l}}}, \frac{1}{\sqrt{y_{l}-1}}\right]$ so that

$$
\begin{aligned}
\mid \frac{2}{\sqrt{y_{l}}+\sqrt{y_{l-1}}}-\frac{1}{\sqrt{\omega_{l}} \mid} & <\frac{1}{\sqrt{y_{l-1}}}-\frac{1}{\sqrt{y_{l}}} \\
& <\frac{1}{\sqrt{a^{2}\left(\omega_{l}\right) \cdot \omega_{l}}}-\frac{1}{\sqrt{\frac{\omega_{l}}{a^{2}\left(\omega_{l}\right)}}} \\
& =\frac{1}{\sqrt{\omega_{l}}}\left(\frac{1}{a\left(\omega_{l}\right)}-a\left(\omega_{l}\right)\right) .
\end{aligned}
$$

If we take $\alpha(\omega)=1-\frac{\varepsilon \sqrt{\omega}}{4}$, then (recalling that $0<\varepsilon, \omega \leq 1$ )

$$
\frac{1}{a(\omega)}-a(\omega)=\frac{2 \varepsilon \sqrt{\omega}-\frac{1}{4} \varepsilon^{2} \omega}{4-\varepsilon \sqrt{\omega}}<\frac{2}{3} \varepsilon \sqrt{\omega} .
$$

Hence,

$$
\left|\frac{2}{\sqrt{y_{l}}+\sqrt{y_{l-1}}}-\frac{1}{\sqrt{\omega_{l}}}\right|<\varepsilon .
$$


Because of the way $\bar{\gamma}$ is defined, the tagged interval $\left(\omega_{0},\left[y_{0}, y_{1}\right]\right)$ cannot have $y_{0}=0$ unless $\omega_{0}=0$. Therefore, when $l=1, h\left(\omega_{1}\right)=h(0)=0$ and $0<y_{1}<\varepsilon^{2}$. Thus,

$$
\begin{aligned}
|S(h, D)-2| & =\left|\sum_{l=2}^{m} \frac{1}{\sqrt{\omega_{l}}}\left(y_{l}-y_{l-1}\right)-2 \sum_{l=1}^{m}\left(\sqrt{y_{l}}-\sqrt{y_{l-1}}\right)\right| \\
& \leq\left|\sum_{l=2}^{m}\left(\frac{1}{\sqrt{\omega_{l}}}-\frac{2}{\sqrt{y_{l}}+\sqrt{y_{l-1}}}\right)\left(y_{l}-y_{l-1}\right)\right|+2 \sqrt{y_{1}} \\
& <\sum_{l=2}^{m} \varepsilon \cdot\left(y_{l}-y_{l-1}\right)+2 \varepsilon<3 \varepsilon .
\end{aligned}
$$

Hence, $h$ is Gauge integrable with $\int_{0}^{1} h=2$.

We shall now note the following basic properties of the Gauge integral.

Theorem 1.1. [5] Assume $h, h_{1}$ and $h_{2}$ are Gauge integrable over $J=[c, d]$, and $h, h_{1}, h_{2}: J \rightarrow \mathbb{R}$

(1) For every $\omega \in \mathbb{R}, \omega h$ is integrable over $J$ with $\int_{J} \omega h=\omega \int_{J} h$.

(2) $h_{1}+h_{2}$ is integrable over $J$ with $\int_{J}\left(h_{1}+h_{2}\right)=\int_{J} h_{1}+\int_{J} h_{2}$.

(3) If $h_{1} \geq 0$ on the interval $J$, then $\int_{J} h_{1} \geq 0$.,

(4) If $h_{1} \geq h_{2}$ on the interval $J$, then $\int_{J} h_{1} \geq \int_{J} h_{2}$.

(5) If $h$ is absolutely integrable over $J$, then $\left|\int_{J} h\right| \leq \int_{J}|h|$.

(6) $h$ is absolutely integrable over $J$ if both $h$ and $|h|$ are integrable over $J$.

(7) Let $c<e<d$. If $h$ is integrable over $[c, e]$ and $[e, d]$, then $h$ is integrable over $[c, d]$, and $\int_{c}^{d} h=\int_{c}^{e} h+\int_{e}^{d} h$

(8) Let $h: J \rightarrow \mathbb{R}$ be integrable over $J$. If $\widetilde{J}$ is a closed subinterval of $J$, then $h$ is integrable over $\widetilde{J}$.

Lemma 1.1. [6] By considering the hypotheses of Henstock's lemma in ([6], page 175), we can write

$$
\sum_{\widehat{P}}\left|h\left(\omega_{i}\right) \cdot \Delta y_{i}-\int_{J_{i}} h\right| \leq 2 \varepsilon
$$

and

$$
\left|\sum_{\widehat{P}}\left(\left|h\left(\omega_{i}\right)\right| \cdot \Delta y_{i}-\left|\int_{J_{i}} h\right|\right)\right| \leq 2 \varepsilon
$$

or, equivalently,

$$
\left|S(|h|, \widehat{P})-\sum_{\widehat{P}}\right| \int_{J_{i}} h|| \leq 2 \varepsilon .
$$

Additionally, we shall consider the definition of bounded variation and properties of $V_{c}^{d} h$.

Definition 1.3. [6] Let $h$ be a function on $[c, d]$, and given a partition $Q=\left\{\left[y_{l-1}, y_{l}\right]\right\}$ of $[c, d]$, the variation of $h$ with respect to $Q$ is

$$
V(h, Q)=\sum_{l}\left|h\left(y_{l}\right)-h\left(y_{l-1}\right)\right| .
$$

The variation of $h$ over $[c, d]$ is

$$
V_{c}^{d} h=\sup V(h, Q)
$$


where the supremum is taken over all partitions $Q$ of $[c, d]$. $h$ is said to be totally bounded variation on $[c, d]$ provided that $V_{c}^{d} h$ is finite, then $B V([c, d])$ will be the set of all such functions.

Lemma 1.2. [6] Assume that $h$ is a function on $[c, d]$. Then

(1) $V(h, P) \leq V(h, Q)$ whenever $P$ and $Q$ are partitions of $[c, d]$ and $Q$ is a refinement of $P$,

(2) If $h \in B V([c, d])$, then $V_{c}^{d} h=V_{c}^{e} h+V_{e}^{b} h$ for any $e \in(c, d)$, and

(3) $V_{c}^{y} h$ and $V_{c}^{y} h-h(y)$ are increasing functions of $y$ on $[c, d]$.

On the other hand, the concept of strongly summable single valued functions were introduced and studied by Borwein in [1]. A nonnegative real-valued Lebesgue measurable function $h(s)$ in the interval $(1, \infty)$ is said to be strongly summable to $L$ if,

$$
\lim _{m \rightarrow \infty} \frac{1}{m} \int_{1}^{m}|h(s)-L| d s=0 .
$$

In [4], Nuray introduced $\lambda$-strongly summable and $\lambda$-statistically convergent functions by taking nonnegative real-valued Lebesgue measurable function on $(1, \infty)$ instead of sequences which are the following:

Definition 1.4. [4] Let $\lambda=\left(\lambda_{u}\right)$ be a non-decreasing sequence of positive numbers tending to $\infty$ such that $\lambda_{u+1} \leq \lambda_{u}+1, \lambda_{1}=1 . \Delta$ denotes the set of all such sequences. For a sequence $y=\left(y_{l}\right)$ the generalized de la Vallée Poussin mean is defined by

$$
t_{u}(y)=\frac{1}{u} \sum_{l \in I_{u}} y_{l},
$$

where $I_{u}=\left[u-\lambda_{u}+1, u\right]$.

Definition 1.5. [4] Let $\lambda \in \Delta$ and $h(s)$ be a real valued function which is measurable in the Lebesgue sense in the interval $(1, \infty)$, if

$$
\lim _{u \rightarrow \infty} \frac{1}{\lambda_{u}} \int_{u-\lambda_{u}+1}^{u}|h(s)-L| d s=0,
$$

then we say that the function $h(s)$ is $\lambda$-strongly summable to $L$. In this case, we can describe $[W, \lambda]-\lim h(s)=L$.

\section{MAIN RESULTS}

In this section, we introduce the concept of $\widetilde{\gamma}$-strongly summable to $L$ with respect to Gauge by considering $h(\vartheta)$ measurable real valued function in the interval $(1, \infty)$. Additionally, using this notion we shall present inclusion theorems to contrast it with other integration techniques.

Definition 2.6. $\delta: J=[c, d] \rightarrow(0, \infty)$ is a positive function, and let us define an open interval valued function on $J$ by setting $\widetilde{\gamma}=\widetilde{\gamma}\left(\omega_{i}\right)=\left(\omega_{i}-\delta\left(\omega_{i}\right), \omega_{i}+\delta\left(\omega_{i}\right)\right)$. If $J_{i}=\left[i-\lambda_{i}+1, i\right]$, we can write $\omega_{i} \in J_{i} \subset \widetilde{\gamma}\left(\omega_{i}\right)$ instead of $\omega_{i}-\delta\left(\omega_{i}\right)<i-\lambda_{i}+1 \leq \omega_{i} \leq i<$ $\omega_{i}+\delta\left(\omega_{i}\right)$. Let $\widetilde{\gamma}=\widetilde{\gamma}\left(\omega_{i}\right) \in \Delta_{G}$, and let $h(\vartheta)$ be a real valued function which is measurable in the Gauge sense on $(1, \infty)$. Provided that $\int h(\vartheta)$ and $\int|h(\vartheta)|$ exist in the Gauge sense and

$$
\lim _{\omega_{i} \rightarrow \infty} \frac{1}{\xi\left(\omega_{i}\right)} \int_{\omega_{i}-\delta\left(\omega_{i}\right)}^{\omega_{i}+\delta\left(\omega_{i}\right)}|h(\vartheta)-L| d \vartheta=0,
$$


where $\xi\left(\omega_{i}\right)=\left(\omega_{i}+\delta\left(\omega_{i}\right)\right)-\left(\omega_{i}-\delta\left(\omega_{i}\right)\right)=2 \delta\left(\omega_{i}\right)$, then we say that the function $h(\vartheta)$ is $\widetilde{\gamma}$-strongly summable to $L$ with respect to Gauge. Whenever this occurs, we can write $[G, \widetilde{\gamma}]-\lim h(\vartheta)=L$.

Let us note that $h:[c, d] \rightarrow \mathbb{R}$ be a function. If $h$ is summable in the Lebesgue sense, then $h$ is strongly summable in the Lebesgue sense, and

$$
\left|\int_{c}^{d} h(y) d y\right| \leq \int_{c}^{d}|h(y)| d y .
$$

This equivalence is not true for the summable with respect to Gauge. To that end, let us consider the following examples,

Example 2.4. Let us define a function

$$
h(\vartheta)=\left\{\begin{array}{l}
(-1)^{l} l, \vartheta \in\left(\frac{1}{l+1}, \frac{1}{l}\right] \\
0, y=0,
\end{array}\right.
$$

is summable in the Gauge sense. However, $h$ is not strongly summable in the Gauge sense because if $|h|$ is not Gauge integrable over $[0,1]$. To see why, suppose that $|h|$ is Gauge integrable over $[0,1]$, then

$$
\int_{0}^{1}|h|=\int_{0}^{\frac{1}{m}}|h|+\int_{\frac{1}{m}}^{1}|h|>\int_{\frac{1}{m}}^{1}|h| .
$$

However, $|h|$ is constant on each of the intervals $\left(\frac{1}{l+1}, \frac{1}{l}\right], 1 \leq l<m$, so that

$$
\int_{\frac{1}{m}}^{1}|h|=\sum_{l=1}^{m-1} \int_{\frac{1}{l+1}}^{\frac{1}{l}}|h|=\sum_{l=1}^{m-1} \frac{1}{l+1} .
$$

Thus, $\int_{1}^{1}|h|$ cannot be finite and $|h|$ is not Gauge integrable. Therefore, $h$ is not strongly summable in the Gauge sense.

Example 2.5. Let us consider a function

$$
h(\vartheta)=\left\{\begin{array}{l}
0, \vartheta=0 \\
2 \vartheta, \cos \left(\frac{\pi}{\vartheta^{2}}\right)+\frac{2 \pi}{\vartheta} \sin \left(\frac{\pi}{\vartheta^{2}}\right), 0<\vartheta \leq 1,
\end{array}\right.
$$

$h$ is Gauge integrable on $[0,1]$ with $\int_{0}^{1} h=-1$. However, $|h|$ is not integrable over $[0,1]$. To see this, let $\tau_{z}=\sqrt{\frac{2}{4 z+1}}$ and $\phi_{z}=\frac{1}{\sqrt{2 z}}$, then the intervals $\left\{\left[\tau_{z}, \phi_{z}\right]\right\}$ are pairwise disjoint and $|h|$ is Gauge integrable over each $\left[\tau_{z}, \phi_{z}\right]$, from the properties of the Gauge Integral, we obtain the following

$$
\int_{\tau_{z}}^{\phi_{z}}|h| \geq\left|\int_{\tau_{z}}^{\phi_{z}} h\right|=\frac{1}{2 z}
$$


If $|h|$ is integrable over $[0,1]$, we have from the finite additivity of the integral

$$
\int_{0}^{1}|h| \geq \sum_{z=1}^{m} \int_{\tau_{z}}^{\phi_{z}}|h| \geq \sum_{z=1}^{m} \frac{1}{2 z}
$$

for every $m$ which is impossible.

As a result, if $h: J \rightarrow \mathbb{R}$ is Gauge integrable over $J$ and $|h|$ is also Gauge integrable over $J$, then $h$ is $\widetilde{\gamma}$-strongly summable in the Gauge sense over $J$. However, If $h$ is Gauge integrable over $J$, but $|h|$ fails to be Gauge integrable over $J$, we say that $h$ is simply $\widetilde{\gamma}$-summable in the Gauge sense over $J$.

Theorem 2.2. Let $J_{i}=\left(\omega_{i}-\delta\left(\omega_{i}\right), \omega_{i}+\delta\left(\omega_{i}\right)\right]$, and $[c, d]=\cup J_{i}$ with $-\infty<c<d<\infty$, and $h: J_{i} \rightarrow \mathbb{R}$ is measurable and $\widetilde{\gamma}$-summable in Gauge sense over $J_{i}$. Let $F(x)=\int_{\omega_{i}-\delta\left(\omega_{i}\right)}^{\omega} h(\vartheta) d \vartheta$ , where $\omega_{i}-\delta\left(\omega_{i}\right)<\omega_{i}<\omega_{i}+\delta\left(\omega_{i}\right)$, be the indefinite integral of $h(\vartheta)$. Then $h(\vartheta)$ is strongly summable in Gauge sense over $J_{i}$ if and only if $F$ is of bounded variation over $J_{i}$.

Proof. Let $h(\vartheta)$ be a function on $J_{i}=\left(\omega_{i}-\delta\left(\omega_{i}\right), \omega_{i}+\delta\left(\omega_{i}\right)\right]$. Given a partition $Q=$ $\left\{\left[i-\lambda_{i}+1, i\right]\right\}$ of $J_{i}$, the total variation of $F$ with respect to $Q$ is

$$
V(F, Q)=\sum_{i}\left|F(i)-F\left(i-\lambda_{i}+1\right)\right| \leq \sum_{i} \int_{i-\lambda_{i}+1}^{i}|h|=\int_{\omega_{i}-\delta\left(\omega_{i}\right)}^{\omega_{i}+\delta\left(\omega_{i}\right)}|h| .
$$

Therefore, $V_{c}^{d} F \leq \int_{c}^{d}|h|<\infty$.

For the converse assume that $F$ is of totally bounded variation of $J_{i}$ and $\varepsilon>0$, there is a partition $P_{0}=\left\{\left[i-\lambda_{i}, i-1\right]\right\}$ such that $V_{\omega_{i}-\delta\left(\omega_{i}\right)}^{\omega_{i}+\delta\left(\omega_{i}\right)} F-\frac{\varepsilon}{2}<V(h, Q) \leq V_{\omega_{i}-\delta\left(\omega_{i}\right)}^{\omega_{i}+\delta\left(\omega_{i}\right)} F$.

Let us define $-\lambda_{i}=\omega_{i}-\delta\left(\omega_{i}\right)-1$ and $i=\omega_{i}+\delta\left(\omega_{i}\right)+1$ and define $\gamma_{0}\left(\omega_{i}\right)$ on $\left[\omega_{i}-\delta\left(\omega_{i}\right), \omega_{i}+\delta\left(\omega_{i}\right)\right]$ by

$$
\gamma_{0}\left(\omega_{i}\right)=\left\{\begin{array}{l}
\left(i-\lambda_{i}, i-1\right), \omega_{i} \in\left(i-\lambda_{i}, i-1\right) \\
\left(i-\lambda_{i}, i\right), \omega_{i}=i-1 .
\end{array}\right.
$$

Then any $\widetilde{\gamma}_{0}-$ fine tagged partition $Q$ will be include $\left\{i-\lambda_{i}, i-1\right\}$ among the tags. Additionally, let us consider $Q$ which is refinement of $P_{0}$. Hence by Lemma 1.2

$$
V_{\omega_{i}-\delta\left(\omega_{i}\right)}^{\omega_{i}+\delta\left(\omega_{i}\right)} F-\frac{\varepsilon}{2}<V\left(h, P_{0}\right) \leq V(h, Q)<V_{\omega_{i}-\delta\left(\omega_{i}\right)}^{\omega_{i}+\delta\left(\omega_{i}\right)} F .
$$

Since $h$ is summable $h$ is integrable, so we can obtain a Gauge $\widetilde{\gamma}_{1}$ such that any $\widetilde{\gamma}_{1}-$ fine tagged partition $P_{2}=\left\{\left(\omega_{i},\left[i-\lambda_{i}-2, i-3\right]\right)\right\}$ that satisfies

$$
\left|\bar{S}\left(h, P_{2}\right)-\int_{\omega_{i}-\delta\left(\omega_{i}\right)}^{\omega_{i}+\delta\left(\omega_{i}\right)} h(\vartheta) d \vartheta\right|<\frac{\varepsilon}{4} .
$$

Lemma 1.1 grants us the following:

$$
\left|S\left(|h|, P_{2}\right)-V\left(F, P_{2}\right)\right|=\left|S\left(|h|, P_{2}\right)-\sum_{i}\right| \int_{i-\lambda_{i}}^{i-1} h(\vartheta) d \vartheta|| \leq \frac{\varepsilon}{2} .
$$


Let $\gamma_{2}(\omega)=\gamma_{0}(\omega) \cap \gamma_{1}(\omega)$ and suppose that $P_{3}$ is any $\tilde{\gamma}-$ fine partition of $\left[\omega_{i}-\delta\left(\omega_{i}\right), \omega_{i}+\delta\left(\omega_{i}\right)\right]$. Then,

$$
\begin{aligned}
& \left|S_{R}\left(|h|, P_{3}\right)-V_{\omega_{i}-\delta\left(\omega_{i}\right)}^{\omega_{i}+\delta\left(\omega_{i}\right)} F\right| \\
\leq & \left|S_{R}\left(|h|, P_{3}\right)-V\left(F, P_{3}\right)\right|+\left|V\left(F, P_{3}\right)-V_{\omega_{i}-\delta\left(\omega_{i}\right)}^{\omega_{i}+\delta\left(\omega_{i}\right)} F\right|<\varepsilon .
\end{aligned}
$$

Hence, $|h|$ is integrable. Since $|h|$ is integrable in the Gauge sense, and $\omega_{i}-\delta\left(\omega_{i}\right)<$ $i-\lambda_{i}+1 \leq \omega_{i} \leq i<\omega_{i}+\delta\left(\omega_{i}\right)$,

$$
\lim _{\omega_{i} \rightarrow \infty} \frac{1}{\xi\left(\omega_{i}\right)} \int_{\omega_{i}-\delta\left(\omega_{i}\right)}^{\omega_{i}+\delta\left(\omega_{i}\right)}|h(\vartheta)-L| d \vartheta=0,
$$

where $\xi\left(\omega_{i}\right)=\left(\omega_{i}+\delta\left(\omega_{i}\right)\right)-\left(\omega_{i}-\delta\left(\omega_{i}\right)\right)=2 \delta\left(\omega_{i}\right)$, then we say that the function $h(\vartheta)$ is $\widetilde{\gamma}_{1}$-strongly summable to $L$ with respect to Gauge.

Theorem 2.3. Let $J_{i}=\left[\omega_{i}-\delta\left(\omega_{i}\right), \omega_{i}+\delta\left(\omega_{i}\right)\right]$ and $[c, d]=\cup J_{i}$ with $-\infty<c<d<\infty$. If $h(\vartheta)$ is $\widetilde{\gamma}$-strongly summable to L with respect to Gauge, then $h(\vartheta)$ is $\widetilde{\gamma}$ - summable to $L$ with respect to Gauge.

Proof. Let $h(\vartheta)$ be a function on $J_{i}=\left[\omega_{i}-\delta\left(\omega_{i}\right), \omega_{i}+\delta\left(\omega_{i}\right)\right]$. Given a partition $P=$ $\left\{\left[i-\lambda_{i}+1, i\right]\right\}$ of $J_{i}$, we are granted from the properties of Gauge integral that $h$ is absolutely integrable over $J_{i}$. Thus, $\left|\int_{J} h\right| \leq \int_{J}|h|$. Since $h(\vartheta)$ is $\widetilde{\gamma}$-strongly summable to $L$ with respect to Gauge, we obtain the following:

$$
\left|\int_{\omega_{i}-\delta\left(\omega_{i}\right)}^{\omega_{i}+\delta\left(\omega_{i}\right)}(h(\vartheta)-L) d \vartheta\right| \leq \int_{\omega_{i}-\delta\left(\omega_{i}\right)}^{\omega_{i}+\delta\left(\omega_{i}\right)}|h(\vartheta)-L| d \vartheta .
$$

Moreover,

$$
\lim _{\omega_{i} \rightarrow \xi\left(\omega_{i}\right) \text { and }\|\Delta T\| \rightarrow 0} \sum_{i} \frac{1}{\xi\left(\omega_{i}\right)} \int_{\omega_{i}-\delta\left(\omega_{i}\right)}^{\omega_{i}+\delta\left(\omega_{i}\right)}|h(\vartheta)-L| d \vartheta=0
$$

where $\|\Delta T\|=\left\|\omega_{i}-\xi\left(\omega_{i}\right)\right\|$. Hence $h(\vartheta)$ is $\widetilde{\gamma}$-summable to $L$ with respect to Gauge.

Theorem 2.4. Let $\lambda=\left(\lambda_{u}\right) \in \Delta, \widetilde{\gamma}=\widetilde{\gamma}\left(\omega_{i}\right) \in \Delta_{G}, J_{i}=\left[\omega_{i}-\delta\left(\omega_{i}\right), \omega_{i}+\delta\left(\omega_{i}\right)\right]$ and $[c, d]=\cup J_{i}$ with $-\infty<c<d<\infty$, and $h(\vartheta)$ be a real valued function in the Gauge sense in the interval $(1, \infty)$, then

1. $[W, \lambda] \subset[G, \widetilde{\gamma}]$

2. If $h(\vartheta)$ is bounded variation and $h$ is $\widetilde{\gamma}$-strongly summable to $L$ with respect to Gauge sense over every measurable subset of $\left[\omega_{i}-\delta\left(\omega_{i}\right), \omega_{i}+\delta\left(\omega_{i}\right)\right]$ (i.e., if $C_{E} f$ is Gauge integrable over $\left[\omega_{i}-\delta\left(\omega_{i}\right), \omega_{i}+\delta\left(\omega_{i}\right)\right]$ for every measurable $\left.E \subset\left[\omega_{i}-\delta\left(\omega_{i}\right), \omega_{i}+\delta\left(\omega_{i}\right)\right]\right)$, then $h$ is $[W]-\lim h(\vartheta)=L$.

Proof. 1. Since all functions are integrable in the Lebesgue sense, they are also integrable in the Gauge sense, if

$$
\frac{1}{\lambda_{u}} \int_{\vartheta \in J_{u}}|h(\vartheta)-L| d \vartheta
$$

exists then

$$
\frac{1}{2 \delta\left(\omega_{i}\right)} \int_{\vartheta \in \widetilde{\gamma}\left(\omega_{i}\right)}|h(\vartheta)-L| d \vartheta
$$


exists. Therefore $[W, \lambda]-\lim h(\vartheta)=L$ implies $[G, \widetilde{\gamma}]-\lim h(\vartheta)=L$.

Then a function $h(\vartheta)=\frac{1}{\vartheta} \sin \frac{1}{\vartheta^{3}}$ is integrable in the Gauge sense, However, it is not integrable in the Lebesgue sense. For instance, $\lim _{\alpha \rightarrow 0} \int_{\alpha}^{1} \frac{1}{\vartheta} \sin \frac{1}{\vartheta^{3}} d \vartheta=\frac{\pi}{6}-\frac{1}{3} \int_{0}^{1} \frac{\sin \vartheta}{\vartheta} d \vartheta$. i.e $h(\vartheta) \notin[W, \lambda]$.

2. If $h(\vartheta)$ be a bounded variation, then clearly $h(\vartheta)$ is bounded. Therefore, we have, since $\frac{4 \delta\left(\omega_{i}\right)}{u} \leq 1$ for all $u$,

$$
\begin{aligned}
\left|\frac{1}{u} \int_{1}^{u}(h(\vartheta)-L) d \vartheta\right| & =\left|\frac{1}{u} \int_{1}^{u-\lambda_{u}}(h(\vartheta)-L) d \vartheta+\frac{1}{u} \int_{\vartheta \in J_{u}}(h(\vartheta)-L) d \vartheta\right| \\
& \leq \frac{1}{u} \int_{1}^{u-\lambda_{u}}|h(\vartheta)-L| d \vartheta+\frac{1}{u} \int_{\vartheta \in J_{u}}|h(\vartheta)-L| d \vartheta \\
& \leq \frac{2}{u} \int_{\vartheta \in J_{u}}|h(\vartheta)-L| d \vartheta \\
& \leq \frac{2}{4 \delta\left(\omega_{i}\right)} \int_{\vartheta \in \widetilde{\gamma}\left(\omega_{i}\right)}|h(\vartheta)-L| d \vartheta=\frac{1}{2 \delta\left(\omega_{i}\right)} \int_{\vartheta \in \widetilde{\gamma}\left(\omega_{i}\right)}|h(\vartheta)-L| d \vartheta .
\end{aligned}
$$

Hence $[W]-\lim h(\vartheta)=L$ since $[G, \widetilde{\gamma}]-\lim h(\vartheta)=L$.

Acknowledgement. The first author is thankful to TUBITAK for a almost two year Visiting Scientist at University of North Florida, Jacksonville, U.S.A. where this work was done during 2017-2019.

\section{REFERENCES}

[1] Borwein, D., Linear Functionals with strongly Cesáro summability, Journal of London Mat. Soc., 40 (1965), 628-634

[2] Henstock, R., Definitions of Riemann Type of Variational Integral, Proc. London Math. Soc., 11 (1961), $402-418$

[3] Kurzweil, J., Generalized Ordinary Differential Equations and Continuous Dependence on a Parameter, Czech. Math. J., 82 (1957), 418-449

[4] Nuray, F., $\lambda$-Strongly summable and $\lambda$-Statistically convergent functions, Iranian Journal of Sci. and Tech., 34 (2010), No. 4, 335-338

[5] Swartz, C., Introduction to Gauge Integrals, World Scientific Publishing Co., 1938

[6] Rosentrater, C. R., Varieties of Integration, Dolciani Mathematical Expositions, 2015

MATHEMATICS DEPARTMENT

SAKARYA UNIVERSITY

SAKARYA, TURKEY

Email address: rabiasavass @hotmail.com

DEPARTMENT OF MATHEMATICS AND STATISTICS

UNIVERSITY OF NORTH FLORIDA

JACKSONVILLE, FLORIDA, USA

Email address: rpatters@unf.edu 